\title{
ESTRESSE E ENFRENTAMENTO EM PROFESSORES: UMA ANÁLISE DA LITERATURA
}

\author{
Kelly Ambrosio Silveira* \\ Sônia Regina Fiorim Enumo** \\ Kely Maria Pereira de Paula*** \\ Elisa Pozzatto Batista****
}

RESUMO: O estresse tem sido associado a problemas de saúde em professores, sendo importante compreender suas estratégias de enfrentamento (coping). Assim, o presente estudo revisou a literatura sobre estresse e coping em professores, com a análise dos principais resultados de pesquisas da área e de suas características metodológicas. Foram analisados artigos publicados entre 2006-2011 em bases internacionais do Portal Periódicos/CAPES, com as palavras-chave: stress, coping e teacher. Os 19 artigos sobre o tema disponíveis integralmente foram analisados. A partir desse material, procedeu-se análise relacionada ao estresse, às estratégias de enfrentamento e às suas implicações para o trabalho docente. É evidenciada a necessidade de práticas avaliativas e interventivas em atenção ao coping e aos sintomas de estresse, de modo a ampliar a compreensão sobre os processos que levem ao bem-estar docente e, consequentemente, à qualidade do ensino ofertado. Palavras-chave: Estresse. Enfrentamento. Revisão de Literatura. Professores.

\footnotetext{
* Doutora em Psicologia pela Universidade Federal do Espírito Santo (UFES). Email: kellyasfs@gmail.com

** Mestre em Educação Especial pela Universidade Federal de São Carlos. Doutora em Psicologia Experimental pelo Instituto de Psicologia da Universidade de São Paulo; Docente do Programa de PósGraduação em Psicologia da PUC-Campinas; Professora emérita da Universidade Federal do Espírito Santo (UFES) e Professora da Pontifícia Universidade Católica de Campinas (PUC-CAMPINAS) e do Programa de Pós-Graduação em Psicologia da Pontifícia Universidade Católica de Campinas (PUC-CAMPINAS). Email:sonia.enumo@puc-campinas.edu.br *** Mestre em Educação pela Universidade do Estado do Rio de Janeir; Doutora em Psicologia pela Universidade Federal Espírito Santo (UFES); Professora da Universidade Federal do Espírito Santo (UFES) e do Programa de Pós-Graduação em Psicologia. Email: kely.paula@ufes.br **** Graduanda em Psicologia pela Universidade Federal do Espírito Santo (UFES). Email: elisapozzatto@gmail.com
} 


\title{
TEACHERS STRESS AND STRUGGLE TO COPE WITH PRESSURE (A LITERATURE ANALYSIS)
}

\begin{abstract}
Stress has been linked to health problems to teachers. Conversely, it is important to understand teachers coping strategies. Thus, this study reviewed the literature on stress and coping to teachers, by analyzing most important researches results in addition to its methodological characteristics. The keywords considered were stress, coping and teacher on articles published by CAPES international portal of journals, between 2006 and 2011. It was possible to examine 19 empirical studies that were fully available. As far as this material is concerned, analyses related to stress, coping strategies and their implications for teaching were conveyed. It showed the need for evaluative and interventionism practices in attention to cope and symptoms of stress, in order to extend the understanding processes that lead to the well-being on teaching and, consequently, the education quality.
\end{abstract}

Keywords: Stress. Coping. Literature Review. Teachers.

\section{INTRODUCุ̃̃O}

Número considerável de professores tem apresentado alterações na saúde física e mental. As alterações estão relacionadas a doenças músculo-esqueléticas (CARDOSO et al., 2009), a problemas com a voz (DELCOR et al., 2004, LUCHESI et al., 2009), ao sono, à memória e ao estresse (GOULART Jr.; LIPP, 2008; POCINHO; CAPELO, 2009; VEDOVATO; MONTEIRO, 2008). Entretanto, os transtornos psíquicos parecem ser a principal causa dos afastamentos do trabalho (GASPARINI; BARRETO; ASSUNÇÃO, 2005). Tal dado é preocupante e coloca em evidência a necessidade de investigação sobre o estresse, pois este pode ser deflagrador dessas disfunções (HANKIN; ABELA, 2005).

$\mathrm{O}$ estresse tem sido analisado no contexto escolar nacional (LIPP, 2002), o que é positivo para a compreensão sobre esse aspecto no trabalho de docentes brasileiros. Além disso, tem sido crescente a participação dos professores como sujeitos de pesquisa sobre essa temática (WITTER, 2003). De modo geral, considera-se o estresse um processo fisiológico resultado de respostas a eventos internos e externos (COMPAS, 2006). Ele é gradativo, partindo de respostas de alerta até a exaustão, no caso de excesso de demandas ou de 
problemas no seu enfrentamento (LIPP, 2003; SELYE, 1983), e pode ocorrer em função de contextos gerais.

O estresse profissional ou ocupacional é entendido como a interação de condições laborais e de características do indivíduo, de tal modo que as exigências que lhe são criadas ultrapassam a capacidade de enfrentamento (GOMES; PEREIRA, 2008). No caso do professor, como a maior parte das vivências no trabalho se dá na relação com o aluno, esta pode ser tanto fonte de estresse, como de recompensas e gratificações (ZAFFARI et al., 2009).

Diferentes fontes de estresse no trabalho docente têm sido apontadas. Entre essas fontes, verificam-se: lidar com incidentes envolvendo comportamento desafiador dos alunos (KELLY et al., 2007), comportamentos inadequados e indisciplina, excesso de trabalho e falta de tempo, diferenças de desenvolvimento e motivação dos alunos e políticas educacionais, sobretudo em professores de séries iniciais do Ensino Fundamental (POCINHO; CAPELO, 2009).

Em casos extremos de estresse no trabalho, pode ocorrer a síndrome de Burnout (BENEVIDES-PEREIRA et al., 2003), um tipo de estresse ocupacional que tende a acometer profissionais envolvidos em atividades de cuidado e atenção direta e altamente emocional (MASLACH; JACKSON, 1986). Esta síndrome é produzida em função de enfrentamentos falhos ou insuficientes (BENEVIDES-PEREIRA, 2002) e de um estado de exaustão física e mental causado pelo excesso de trabalho, em sobrecarga e intensidade. Está associada às dimensões exaustão emocional (fadiga e perda de recursos emocionais, como esgotamento emocional devido ao trabalho), despersonalização (indiferença e atitudes distantes para com o trabalho realizado) e baixa realização profissional (comprometimento da eficácia percebida no desenvolvimento do trabalho) (MASLACH; JACKSON, 1986). Em consequência dessas características, os processos interativos podem ser prejudicados devido ao trato impessoal no ambiente de trabalho, voltado também para os alunos (YAEGASHI et al., 2008).

Com isso, deve ser considerada a análise das alternativas para a minimização do estresse docente, incluindo tanto as demandas do trabalho quanto os processos de regulação. A regulação frente ao estresse está relacionada às respostas comportamentais e emocionais, sejam elas falhas ou não, e inclui variáveis sociais e culturais, sendo, desse modo, um processo ativo (ALDWIN, 2007). Inclui processos 
de coping, ou de enfrentamento, entendidos como "[...] modos de mobilizar, guiar, manejar e direcionar o comportamento, a emoção e a orientação sob condições estressantes [...]" (SKINNER; ZIMMER-GEMBECK, 2007, p. 122). Lazarus e Folkman (1984) concebem esse construto como respostas voluntárias ao estresse, a partir de esforços conscientes para a regulação da emoção ou para a resolução de problemas. A partir dessas premissas, outras definições têm sido adaptadas.

Outros pesquisadores têm partido de outras perspectivas, com foco na regulação do comportamento. Compas (2006), por exemplo, considera as implicações do coping na regulação da cognição, do comportamento e do ambiente a partir de estratégias que envolvem: controle primário para a solução de problemas, expressão emocional e modulação emocional; adaptação, ligada à aceitação, distração, reestruturação cognitiva e pensamento positivo; e retraimento, que compreende evitação, negação e pensamento mágico.

A partir de uma ampla revisão crítica sobre este tema, incluindo recursos avaliativos publicados entre a década de 1980 e o ano 2000, Skinner et al. (2003) identificaram quatrocentas categorias, com diferentes definições e classificações sobre o coping. Esses autores propuseram um modelo hierárquico do processo de enfrentamento, pelo qual se verificam instâncias ou comportamentos de coping, relacionados às estratégias de enfrentamento, e famílias de coping, ou conjuntos de estratégias com funcionalidade similar, visando à adaptação geral do indivíduo. Este modelo apresenta o enfrentamento como aspecto integrante da regulação comportamental frente a processos motivacionais. Assim, trata-se de um construto em pleno desenvolvimento.

Os conceitos de estresse e de coping, em seus variados arranjos, têm sido aplicados em várias áreas, incluindo a organizacional (MURTA; TROCCOLI, 2004). Nesta, considerase que características do ambiente, como o conflito de papéis, e características pessoais, como a autoeficácia percebida no trabalho, podem definir o tipo de enfrentamento. A autoeficácia, por sua vez, refere-se a quanto uma pessoa acredita que pode controlar ou modificar o ambiente (BANDURA, 1997). Assim, professores com maior senso de autoeficácia podem identificar maior possibilidade de adaptações em seu próprio comportamento diante das necessidades observadas (ALMOG; SHECHTMAN, 2007). 
Se os funcionários acreditam que a situação pode mudar ou que podem atuar de modo a diminuir a extensão dos problemas vivenciados, tendem a utilizar estratégias baseadas na solução de problemas. Ao contrário, podem ser adotadas respostas emocionais ou de esquiva (BOYD; LEWIN; SAGER, 2009).

Sabe-se, em complemento, que algumas variáveis podem modular a experiência de estresse e a utilização de estratégias de enfrentamento. Professores com menos tempo de serviço, por exemplo, podem apresentar maior vulnerabilidade ao estresse e mais estratégias de enfrentamento baseadas na emoção (GOMES; PEREIRA, 2008). Aqueles com menos estratégias de controle e mais esquiva frente aos estressores também podem apresentar maior vulnerabilidade ao estresse (POCINHO; CAPELO, 2009). Entre os mais jovens, pode haver mais estresse e uso mais frequente de estratégias ligadas ao retraimento social (ALVES; OLIVEIRA, 2008). Além disso, sabe-se que a exaustão emocional associa-se ao uso mais frequente do foco na emoção e desligamento mental (CARLOTTO; CÂMARA, 2008).

Considera-se importante o desenvolvimento de mais estudos sobre as relações entre processos de estresse e de coping no trabalho docente. Como as terminologias e as técnicas avaliativas têm sido diversificadas, torna-se útil a análise da literatura internacional sobre a condução das pesquisas e os seus principais resultados. Assim, pode-se ampliar a compreensão desses conceitos no cenário escolar e a sua aplicabilidade em pesquisas e intervenções de âmbito nacional.

Este estudo pretendeu verificar, a partir da literatura científica internacional publicada entre os anos de 2006 a 2011, o conteúdo de artigos empíricos que tratavam de relações entre o estresse e o coping em professores. Pretendeu-se identificar os principais aspectos metodológicos e os resultados ligados ao estresse, às estratégias de enfrentamento e às implicações do estresse e do coping no trabalho docente. As sugestões de intervenção para a promoção do coping e diminuição do estresse também foram abordadas.

\section{MÉTODO}

Foi realizado um estudo de revisão da literatura (MOREIRA, 2004). Os artigos pesquisados integravam o acervo de artigos internacionais publicados entre os anos de 2006 e 2011 em bases 
de dados indexadas no Portal Periódicos CAPES/MEC. As bases de dados consultadas foram: PsycINFO (APA), Academic Search Premier (ASP) (EBSCO), Cambridge Journals Online, Education Full Text (Wilson), JSTOR Arts \& Sciences Collection, Oxford Journals (Oxford University Press), Project Muse, PsycArticles (APA), SAGE Journals Online, SocINDEX with Full Text (EBSCO), SpringerLink (MetaPress) e Wiley Online Library. A busca foi efetuada a partir de palavras-chave utilizadas isoladamente e em conjunto: stress, teacher e coping.

Foram resgatados 595 resumos. A análise preliminar do título e do resumo resultou na exclusão de estudos de temática diferente, restando 36 artigos. Desses, 17 artigos não estavam acessíveis em sua integralidade para a consulta, de modo que foram excluídos do processo, resultando, assim, 19 artigos para análise.

A análise qualitativa dos dados atendeu a categorias sobre atributos dos artigos: ano de publicação, local de realização, objetivos, participantes, tipo de estudo (qualitativo, quantitativo ou misto), delineamento (descritivo, correlacional ou experimental), coleta de dados (observação naturalística, administração de instrumentos de autorrelato e entrevista), instrumentos e resultados ligados aos eventos estressores, às diferenças individuais no estresse e no coping e às suas implicações no trabalho docente. As sugestões expressas sobre intervenção nesse contexto também foram abordadas.

\section{RESULTADOS E DISCUSSÃO}

Os estudos contemplaram análises sobre estresse, burnout, estilos de coping, relações entre essas variáveis e características pessoais, como idade, autoeficácia e características laborais, a exemplo do tempo de serviço e da modalidade de ensino. A avaliação de programa interventivo e a análise da validade de instrumento também foram identificadas. Os objetivos dos estudos encontram-se na Tabela 1.

As publicações dos anos de 2008 e 2009 apresentaram maior frequência entre os artigos (seis e cinco artigos, respectivamente). Os Estados Unidos da América foram o país mais representado (cinco artigos), seguido da Alemanha e China (três artigos, cada). Não houve nenhum estudo brasileiro indexado nas bases pesquisadas com esta temática (Tabela 2). 
TABELA 1 - OBJETIVOS DOS 19 ARTIGOS SOBRE ESTRESSE E COPING DOCENTE

\begin{tabular}{|c|}
\hline Descrição dos objetivos - o que foi investigado? \\
\hline $\begin{array}{l}\text { Relaç̃oes entre estressores percebidos e respostas de coping de professores de classes especiais e } \\
\text { inclusivas (ANTONIOU; POLYCHRONI; KOTRONI, 2009). }\end{array}$ \\
\hline Relações entre coping e percepção de controle sobre os alunos (BALOGLU, 2008). \\
\hline $\begin{array}{l}\text { Relaç̃os preditivas entre estressores, coping e autoeficácia sobre o burnout (DOMÉNECH- BETORET; } \\
\text { ARTIGA, 2010). }\end{array}$ \\
\hline Relações entre comparações com colegas, estilos de coping e burnout (CARMONA et al., 2006). \\
\hline Relações entre coping, inteligência emocional e autoeficácia percebida (CHAN, 2008). \\
\hline $\begin{array}{l}\text { Padrões voltados à percepção de comportamento disruptivo de alunos, regulação da emoção, coping } \\
\text { e burnout (CHANG, 2009). }\end{array}$ \\
\hline Efeitos de um treinamento de atenção autodirigida sobre o estresse (GOLD et al. 2010). \\
\hline Os modos como os professores utilizam os espaços de trabalho para enfrentar o estresse (GULWADI, 2006). \\
\hline Associação entre demandas estressoras, engajamento e exaustão (KLUSMANN et al., 2008). \\
\hline $\begin{array}{l}\text { Relaç̃ões preditivas entre padrões autorregulatórios e exaustão emocional sobre a performance } \\
\text { instrucional (KLUSMANN et al. 2008). }\end{array}$ \\
\hline Características psicométricas de instrumento sobre estresse e coping docente (LAMBERT et a/2009). \\
\hline $\begin{array}{l}\text { Relaç̃oes entre dados sociodemográficos, autoeficácia percebida, suporte social e estratégias de } \\
\text { enfrentamento (LAUGAA et al., 2008). }\end{array}$ \\
\hline $\begin{array}{l}\text { Indicadores de estresse em professores após intervenção para redução do estresse (LHOSPITAL; } \\
\text { GREGORY, 2009). }\end{array}$ \\
\hline $\begin{array}{l}\text { Causas do estresse e modos de enfrentamento em professores iniciantes na carreira (RIEG; } \\
\text { PAQUETTE; CHEN, 2007). }\end{array}$ \\
\hline Dados sobre estresse e coping em professores (SHARPLIN; O'NEILL; CHAPMAN, 2011) \\
\hline Relaç̃os entre perfeccionismo, estresse e coping (SHEN, 2009). \\
\hline Relaç̃oes entre estresse e variáveis pessoais e do trabalho (STOEBER; RENNERT, 2008). \\
\hline $\begin{array}{l}\text { Relações preditivas entre pressão, satisfação no trabalho e o coping sobre a saúde mental (ZURLO; } \\
\text { PES; COOPER, 2007). }\end{array}$ \\
\hline Relações entre rejeição de alunos, despersonalização e coping (WONG; WAl; ROSEN, 2007). \\
\hline
\end{tabular}


TABELA 2 - CARACTERÍSTICAS DOS ARTIGOS RESGATADOS

\begin{tabular}{|c|c|c|c|}
\hline Autores & Local & Participantes & Tipo \\
\hline Antoniou et al. (2009) & Grécia & 158 professores de educação especial & $\begin{array}{l}\text { Quantitativo, } \\
\text { Correlacional }\end{array}$ \\
\hline Baloglu (2008) & Turquia & 267estudantes de graduação & $\begin{array}{l}\text { Quantitativo, } \\
\text { Correlacional }\end{array}$ \\
\hline $\begin{array}{l}\text { Doménech-Betoret; } \\
\text { Artiga (2010) }\end{array}$ & Espanha & 724 professores primários e secundários & $\begin{array}{l}\text { Quantitativo, } \\
\text { Correlacional }\end{array}$ \\
\hline Carmona et al. (2006) & Espanha & $\begin{array}{l}558 \text { professores de escolas primárias e } \\
\text { secundárias }\end{array}$ & $\begin{array}{l}\text { Quantitativo, } \\
\text { Correlacional }\end{array}$ \\
\hline Chan (2008) & China & 273 professores recém-graduados & $\begin{array}{l}\text { Quantitativo, } \\
\text { Correlacional }\end{array}$ \\
\hline Chang (2009) & $\begin{array}{l}\text { Estados } \\
\text { Unidos }\end{array}$ & 555 professores iniciantes de carreira & $\begin{array}{l}\text { Quantitativo, } \\
\text { Correlacional }\end{array}$ \\
\hline Gold et al. (2010) & Inglaterra & 11 professores primários & $\begin{array}{l}\text { Quantitativo, } \\
\text { Pré-experimental }\end{array}$ \\
\hline Gulwadi (2006) & $\begin{array}{l}\text { Estados } \\
\text { Unidos }\end{array}$ & 71 professores de ensino fundamental & $\begin{array}{l}\text { Quantitativo, } \\
\text { Descritivo }\end{array}$ \\
\hline Klusmann et al. (2008) & Alemanha & 1939 professores secundários & $\begin{array}{l}\text { Quantitativo, } \\
\text { Correlacional }\end{array}$ \\
\hline Klusmann et al. (2008) & Alemanha & 1789 professores de matemática & $\begin{array}{l}\text { Quantitativo, } \\
\text { Correlacional }\end{array}$ \\
\hline Lambert et al. (2009) & $\begin{array}{l}\text { Estados } \\
\text { Unidos }\end{array}$ & 521 professores de ensino fundamental & $\begin{array}{l}\text { Quantitativo, } \\
\text { Correlacional }\end{array}$ \\
\hline Laugaa et al. (2008) & França & 410 professores de ensino fundamental & $\begin{array}{l}\text { Quantitativo, } \\
\text { Correlacional }\end{array}$ \\
\hline $\begin{array}{l}\text { Lhospital; Gregory } \\
\text { (2009) }\end{array}$ & $\begin{array}{l}\text { Estados } \\
\text { Unidos }\end{array}$ & 33 professores de ensino fundamental & $\begin{array}{l}\text { Quantitativo, } \\
\text { Experimental }\end{array}$ \\
\hline Rieg et al. (2007) & $\begin{array}{l}\text { Estados } \\
\text { Unidos }\end{array}$ & 39 Professores de universidade rural & $\begin{array}{l}\text { Misto, } \\
\text { Descritivo }\end{array}$ \\
\hline Sharplin et al. (2011) & Austrália & $\begin{array}{l}29 \text { professores designados para áreas } \\
\text { rurais }\end{array}$ & $\begin{array}{l}\text { Qualitativo, } \\
\text { Descritivo }\end{array}$ \\
\hline Shen (2009) & China & $\begin{array}{l}530 \text { professores ensino fundamental e } \\
\text { secundário }\end{array}$ & $\begin{array}{l}\text { Quantitativo, } \\
\text { Correlacional }\end{array}$ \\
\hline Stoeber; Rennert (2008) & Alemanha & 118 professores secundários & $\begin{array}{l}\text { Quantitativo, } \\
\text { Correlacional }\end{array}$ \\
\hline Zurlo et al. (2007) & Itália & $\begin{array}{l}320 \text { professores educação infantil, } \\
\text { fundamental e secundário }\end{array}$ & $\begin{array}{l}\text { Quantitativo, } \\
\text { Correlacional }\end{array}$ \\
\hline Wong et al. (2007) & China & $\begin{array}{l}1010 \text { professores de educação infantil } \\
\text { recém-graduados }\end{array}$ & $\begin{array}{l}\text { Quantitativo, } \\
\text { Correlacional }\end{array}$ \\
\hline
\end{tabular}


As pesquisas quantitativas apresentaram maior frequência (17 artigos) em comparação às pesquisas qualitativas (um) ou mistas (um). As amostras variaram em total de participantes (11 a 1.939) e em modalidade de ensino. Apesar de os sistemas de ensino variarem entre os países, as séries equivalentes ao ensino fundamental brasileiro foram as mais frequentemente representadas, compondo amostras exclusivas em quatro estudos. As análises de tipo correlacional predominaram (14 artigos), em comparação às análises estritamente descritivas (3) ou de caráter experimental (2) (Tabela 2).

Os artigos contaram com questionários sociodemográficos. Contudo, apenas dois estudos utilizaram entrevistas como técnica para a coleta de dados. Num estudo, a coleta foi efetuada unicamente a partir de um questionário especialmente elaborado. Por outro lado, em 17 artigos, foram utilizados instrumentos avaliativos com base no autorrelato e publicados previamente. Estes se referiram a temas como autoeficácia, inteligência emocional, perfeccionismo, regulação emocional, habilidades mentais, saúde mental, depressão, ansiedade e locus de controle.

Sobre o estresse, foram identificados seis instrumentos, com igual frequência; entre eles, o Sources of Pressure in Teaching Scale (TRAVERS; COOPER, 1996). Esses instrumentos, em geral, fazem o mapeamento de condições potencialmente estressantes para os professores. Para a avaliação do burnout, um único instrumento foi utilizado, o Maslach Burnout Inventory - MBI (MASLACH; JACKSON, 1986) e suas adaptações, com aplicação em seis estudos.

Em atenção ao coping, foram utilizados seis instrumentos, com maior frequência para o Ways of Coping Questionnaire (FOLKMAN; LAZARUS, 1988) e suas adaptações, presentes em cinco artigos. Este instrumento é representativo de uma linha cognitivista sobre a relação estresse e coping e enfatiza seu caráter transacional. Outros instrumentos utilizados também apresentam semelhanças com esta base conceitual, apesar de integrarem um diferente arranjo de categorias, a exemplo do Occupational Stress Indicator (COOPER, SLOAN, WILLIAMS, 1988) (Tabela 3). 
TABELA 3 - INSTRUMENTOS PARA A AVALIAC̣ÃO DE ESTRESSE, BURNOUTE COPING PRESENTES NOS ARTIGOS

\begin{tabular}{|c|c|c|}
\hline Construto avaliado/Instrumento & $f$ & Características/Dimensões \\
\hline \multicolumn{3}{|l|}{ Estresse } \\
\hline $\begin{array}{l}\text { Multicontext Stressors Scale } \\
\text { (DOMÉNECH-BETORET, 2006) }\end{array}$ & 1 & $\begin{array}{l}\text { Problemas motivacionais e comportamentais dos } \\
\text { alunos, Pouca participação nas decisões, Ambiguidade } \\
\text { nas decisões administrativas, Diversidade entre os } \\
\text { estudantes, Excesso de trabalho, Pouco envolvimento } \\
\text { dos pais. }\end{array}$ \\
\hline $\begin{array}{l}\text { Depression Anxiety Stress Scales } \\
\text { (HENRY, CRAWFORD 2005) }\end{array}$ & 1 & Estresse, Depressão e Ansiedade. \\
\hline $\begin{array}{l}\text { Standard Questionnaire-Teacher Stress } \\
\text { (EVERAERT, 2003) }\end{array}$ & 1 & $\begin{array}{l}\text { Comportamentos hiperativos, comportamentos sociais } \\
\text { positivos, interferência na dinâmica da aula, apoio de } \\
\text { colegas e apoio de pais e comunidade. }\end{array}$ \\
\hline $\begin{array}{l}\text { Index of Teaching Stress (ABIDIN; } \\
\text { GREENE; KONOLD, 2004) }\end{array}$ & 1 & $\begin{array}{l}\text { Estresse diádico, dificuldades ligadas a um aluno em } \\
\text { particular. }\end{array}$ \\
\hline $\begin{array}{l}\text { Sources of Pressure in Teaching Scale } \\
\text { (TRAVERS, COOPER, 1996) }\end{array}$ & 1 & $\begin{array}{l}\text { Acúmulo de trabalho, problemas no status profissional, } \\
\text { reconhecimento e suporte no trabalho por colegas, } \\
\text { alunos e seus pais. }\end{array}$ \\
\hline $\begin{array}{l}\text { Classroom Appraisal of Resources and } \\
\text { Demands (LAMBERT; MCCARTHY; } \\
\text { ABBOTT-SHIM, 2001) }\end{array}$ & 1 & Demandas estressoras da classe e recursos da classe. \\
\hline \multicolumn{3}{|l|}{ Burnout } \\
\hline $\begin{array}{l}\text { Maslach Burnout Inventory (MASLACH, } \\
\text { JACKSON, 1986) }\end{array}$ & 6 & $\begin{array}{l}\text { Exaustão emocional, despersonalização e baixa } \\
\text { realização profissional. }\end{array}$ \\
\hline \multicolumn{3}{|l|}{ Coping } \\
\hline $\begin{array}{l}\text { Ways of Coping Questionnaire } \\
\text { (FOLKMAN; LAZARUS, 1988) }\end{array}$ & 5 & $\begin{array}{l}\text { Coping baseado no problema e coping baseado na } \\
\text { emoção. Versões contendo Solução de problemas, } \\
\text { evitação, busca de suporte e pensamento desejoso. } \\
\text { Redução para coping ativo (solução de problemas) e } \\
\text { passivo (evitativo). }\end{array}$ \\
\hline $\begin{array}{l}\text { Erfurt Stress Inventory for Teachers } \\
\text { (BOHM-KASPER et al., 2000) }\end{array}$ & 1 & Coping ativo e evitativo. \\
\hline $\begin{array}{l}\text { Occupational Stress Indicator (COOPER, } \\
\text { SLOAN, WILLIAMS, 1988) }\end{array}$ & 3 & $\begin{array}{l}\text { Coping ativo e paliativo, ligados ao suporte social, } \\
\text { lógica, envolvimento, foco na tarefa, manejo do tempo e } \\
\text { suporte na tarefa. }\end{array}$ \\
\hline $\begin{array}{l}\text { COPE Inventory (CARVER; SCHEIER; } \\
\text { WEINTRAUB, 1989) }\end{array}$ & 2 & $\begin{array}{l}\text { Reinterpretação positiva, Desengajamento mental e } \\
\text { comportamental, Foco na emoção, Uso de suporte social } \\
\text { e instrumental, Uso de suporte emocional, Afastamento, } \\
\text { Coping ativo, Coping religioso, Negação, Humor, Uso } \\
\text { de Substância, Aceitação, Planejamento, Supressão de } \\
\text { atividades concorrentes. }\end{array}$ \\
\hline $\begin{array}{l}\text { Occupational Stress and Coping Inventory } \\
\text { (SCHAARSCHMIDT, FISCHER, 1997) }\end{array}$ & 2 & $\begin{array}{l}\text { Significância subjetiva do trabalho, distanciamento } \\
\text { emocional, coping ativo e estabilidade mental. }\end{array}$ \\
\hline $\begin{array}{l}\text { Questionário aberto para respostas de } \\
\text { coping (STRAUSS, CORBIN, 1998) }\end{array}$ & 1 & $\begin{array}{l}\text { Estratégias executadas interna e externamente, com } 0 \\
\text { apoio do ambiente. }\end{array}$ \\
\hline
\end{tabular}

Fonte: Dados da pesquisa. 
Os resultados foram agrupados, inicialmente, considerandose os fatores estressantes e as diferenças individuais nas respostas de estresse $e$ de enfrentamento.

Os fatores estressantes no contexto escolar foram: lidar com a classe e manter a disciplina, aplicar as tarefas, organizar grupos de trabalho, ajudar crianças com problemas comportamentais, comunicar conceitos e preparar recursos para lições (RIEG; PAQUETTE; CHEN, 2007). Foram apontados problemas organizacionais, como a falta de recursos e equipamentos, o excesso de responsabilidade pelos alunos, o pouco suporte do governo e pressões de tempo na escola (ANTONIOU; POLYCHRONI; KOTRONI, 2009), o maior número de classes regidas (KLUSMANN et al., 2008), a alta carga de trabalho (DOMÉNECH-BETORET; ARTIGA, 2010), o pouco suporte profissional, excesso de trabalho, as salas cheias, a falta de apoio dos pais dos alunos, o comportamento agressivo e a falta de suporte (ZURLO; PES; COOPER, 2007). A quantidade acumulada de problemas comportamentais, criando novos desafios (LHOSPITAL; GREGORY, 2009), o mau comportamento, a baixa motivação dos alunos, bem como o envolvimento insuficiente dos pais (DOMÉNECH-BETORET; ARTIGA, 2010) também foram características associadas a maior estresse. Ser desprezado também pode estar ligado ao estresse, com impacto na despersonalização (WONG; WAI; ROSEN, 2007). Além disso, a pressão conjunta dos pais, professores e alunos, quando percebida, acompanha mais avaliações de perigo e de perda (STOEBER; RENNERT, 2008).

As diferenças individuais nas respostas de estresse foram apontadas por Klusmann et al. (2008) como responsáveis pela maior variabilidade para a experiência emocional e motivacional, quando comparadas a variáveis do trabalho escolar. Em adição, o esforço para manter o comportamento perfeito, com reação negativa após erros, tem sido uma característica pessoal com implicações para o estresse (STOEBER;RENNERT,2008), com destaque para a maior associação entre as preocupações perfeccionistas e o burnout, quando comparadas à busca por perfeição. Professores que se identificam com colegas mal-sucedidos no desenvolvimento de suas tarefas também tendem a experienciar mais burnout (CARMONA et al., 2006).

Ainda sobre as características individuais, verificou-se que a emoção negativa associa-se à maior intensidade de burnout (CHANG, 2009) e que os fatores de personalidade atuam como 
moderadores entre condições ambientais e reações emocionais e motivacionais (KLUSMANN et al., 2008).

A idade dos professores foi outro fator apontado, sendo a faixa entre 41-50 anos associada a mais estresse do que entre os mais jovens e mais velhos (ANTONIOU; POLYCHRONI; KOTRONI, 2009). Diferenças de gênero no estresse também foram observadas. Professoras tendem a apresentar mais exaustão emocional, que aumenta com a experiência (DOMÉNECH-BETORET; ARTIGA, 2010) e também reportam mais estresse relacionado à responsabilidade com os alunos, pressões de tempo na escola e falta de motivação dos alunos, ao passo que os homens relatam estresse acerca de dificuldades com o comportamento dos alunos (ANTONIOU; POLYCHRONI; KOTRONI, 2009) e sofrem mais despersonalização (DOMÉNECH-BETORET; ARTIGA, 2010). Por outro lado, os homens utilizaram estratégias de enfrentamento ligadas à gestão de tempo, pensar sobre a situação, traçar objetivos e planejar o futuro (ANTONIOU; POLYCHRONI; KOTRONI, 2009), ou seja, de modo geral, utilizaram ações de aproximação, ativas, voltadas para a solução dos problemas.

Em relação à idade e às estratégias de enfrentamento, Betoret, Doménech e Artiga (2010) verificaram que a frequência do coping ativo diminui com a experiência profissional. Também houve diferença de idade em relação ao uso das estratégias de enfrentamento. Professores com idade entre 41-50 anos costumavam usar estratégias de suporte social, reorganização do trabalho e lidar com os problemas imediatamente (LAUGAA; RASCLE; BRUCHONSCHWEITZER, 2008).

O papel da autoeficácia foi associado ao coping, tendo efeito positivo (DOMÉNECH-BETORET; ARTIGA, 2010), sobretudo no coping ativo, quando associado a altos escores em inteligência emocional (CHAN, 2008) e ao suporte social (SHEN, 2009). Além disso, a autoeficácia está ligada à percepção de que os objetivos serão alcançados, levando ao coping ligado à solução de problemas, com comportamentos como racionalizar, analisar a situação, pensar sobre aspectos positivos e encorajar os alunos (LAUGAA; RASCLE; BRUCHON-SCHWEITZER, 2008). Professores com mais autoeficácia dão mais atenção às oportunidades que facilitam o coping frente ao estresse. Em contrapartida, pouca autoeficácia percebida pode levar à predominância de estratégias baseadas na emoção (SHEN, 2009). 
Considerando, agora, as implicações do estresse e do coping no trabalho docente, foram agregadas informações sobre o impacto dos recursos ambientais e individuais sobre o trabalho e sobre o efeito das estratégias de enfrentamento.

O impacto dos recursos ambientais e individuais sobre o trabalho docente foi observado por Zurlo, Pes e Cooper (2007). Eles observaram que a satisfação com o trabalho, na ausência de suporte social, pode levar a uma postura mais individualista, gerando, no longo prazo, um efeito negativo na qualidade do ensino. É, portanto, um fator de risco para estresse. Entre os recursos ambientais, verificou-se uma correlação positiva entre as barreiras pedagógicas e o burnout (DOMÉNECH-BETORET; ARTIGA, 2010). Nos casos de poucos recursos, em que se percebem mais as demandas ambientais, a percepção de pouca competência/ eficácia levaria ao uso de estratégias de escape da situação estressora (GULWADI, 2006). Além disso, Stoeber e Rennert (2008) verificaram que, quando é percebida pressão conjunta dos pais, dos professores e dos alunos, há mais avaliações de perigo e perda, com coping evitativo e menor comprometimento no trabalho.

Ainda, professores que utilizam coping ativo com mais frequência para superar as barreiras pedagógicas sentem-se mais eficazes no seu trabalho e relatam menos despersonalização, modulando a relação entre as barreiras pedagógicas e o burnout (DOMÉNECH-BETORET; ARTIGA, 2010). Lhospital e Gregory (2009) constataram que, quando o professor auxilia na resolução das dificuldades do aluno, seu senso de eficácia aumenta, protegendo-o do estresse. Em contrapartida, os problemas emocionais de alunos podem enfraquecer o senso de eficácia docente e levar a uma atitude mais crítica do professor em relação às suas próprias habilidades (LAMBERT et al., 2009). Deve-se considerar também a possibilidade de associação entre o maior estresse percebido e a menor despersonalização, sugerindo que o professor mais sensível e atento às dificuldades do estudante está mais propenso ao estresse (LAUGAA; RASCLE; BRUCHON-SCHWEITZER, 2008).

Em alguns contextos, a evitação pode ser apropriada para o bem-estar no trabalho, ajustando o escape de lugares e problemas, o que geraria menos preocupações, tal como observado por Sharplin, O'neill e Chapman, (2011) na análise de professores rurais. Além disso, professores com mais coping focalizado no problema e menos 
na emoção são menos resistentes às mudanças do cotidiano escolar (WONG; WAI; ROSEN, 2007). Em adição, professores mais engajados e resilientes parecem estar mais atentos às necessidades individuais dos alunos (KLUSMANN et al., 2008). Contudo, parece não haver uma relação direta entre autorregulação dos professores e sucesso dos alunos (KLUSMANN et al., 2008).

Os efeitos do coping sobre a docência localizam-se também na avaliação das dificuldades dos alunos (LHOSPITAL; GREGORY, 2009). Além disso, estimulam a experiência motivacional destes, a partir de estimulação cognitiva e de suporte social fornecido pelo professor (KLUSMANN et al., 2008). Ainda sobre essa temática, percebeu-se que, quanto maior o estresse e burnout, há menos criação de ambientes sociais positivos para as crianças. Com isso, há mais modelos para comportamentos sociais negativos, principalmente em casos de exaustão emocional e de percepção do aluno como objeto, em vez de sujeito em desenvolvimento (LAMBERT et al., 2009). A dificuldade na interação com alunos pode surgir pelo fato de os professores não terem oportunidades de aprender estratégias para lidar com o estresse ou para se preparar para a profissão (BALOGLU, 2008).

Considerando, por último, os dados sobre propostas de intervenção para a promoção do coping e diminuição do estresse, identificaram-se justificativas e sugestões para intervenções e efeitos de treinamentos.

Lhospital e Gregory (2009) afirmaram a importância de suporte ao professor por meio de apoio, estrutura e auxílio em momentos de crise. O treinamento em mecanismos de enfrentamento, por sua vez, auxiliaria na conexão emocional e social, na identificação de estressores (RIEG; PAQUET'TE; CHEN, 2007) e no aprimoramento da resiliência e no desenvolvimento profissional (SHARPLIN; O’NEILL; CHAPMAN, 2011).

A partir da consideração de Klusmann et al. (2008) de que a ausência de sintomas de burnout não significa, necessariamente, alto engajamento com o trabalho, e de que mesmo professores engajados podem estar passando por exaustão emocional, faz-se necessária a proposição de programas preventivos e interventivos para professores, voltados para a qualidade de vida e do trabalho docente.

As sugestões de objetivos das intervenções foram diversas. Visavam auxiliar nos julgamentos e na regulação da emoção e do desempenho frente aos problemas comportamentais de alunos (CHANG, 2009); desenvolver coping mais facilitador frente a situações 
estressoras (SHEN, 2009); aplicar programas de manejo emocional (CHAN, 2008); treinar a atenção para atitudes que levem ao bem-estar pessoal, reduzir dificuldades interpretativas, melhorar metas pessoais e aumentar a capacidade para lidar com as demandas educacionais (GOLD et al., 2010); treinar o uso coping focalizado no problema, diminuindo os efeitos do desprezo e da despersonalização para lidar com a rejeição (WONG; WAI; ROSEN, 2007).

Laugaa, Rascle e Bruchon-Schweitzer (2008) propuseram três tipos de intervenções com foco no manejo do estresse e do coping docente: as primárias, as secundárias e as terciárias. As intervenções primárias centram-se na organização do trabalho para a redução de estressores e aumento de recursos pessoais, afeto pelo trabalho e autoeficácia, a partir da reestruturação do trabalho e das relações interpessoais. As intervenções secundárias refinam as estratégias de enfrentamento de dois modos, incluindo a identificação de estratégias para resolver os problemas e reduzir tensão emocional em situação estressora. A análise coletiva das situações de trabalho e das possibilidades de solução dos problemas, com foco na diminuição do isolamento e da culpa, por meio de suporte mútuo, seria a outra possibilidade. As intervenções terciárias minimizam sintomas de estresse manifestos.

As intervenções, assim, podem melhorar a qualidade de vida dos professores, a partir da troca de informações sobre as atividades laborais e do manejo de condições potencialmente estressoras; do suporte para o desenvolvimento de competências e habilidades e da estabilidade a partir da atenção a estratégias de enfrentamento e resiliência para a promoção da qualidade no trabalho.

As discussões sobre os efeitos de treinamento nessa perspectiva também foram variadas. Lhospital e Gregory (2009) afirmaram que professores podem apresentar menos distress relacionado às necessidades das crianças, a partir de suporte de equipe especializada. Por sua vez, Gold et al. (2010) afirmaram que os professores podem experimentar uma diminuição de estresse emocional, a partir da estimulação das habilidades autorreferidas de motivação, autoconfiança, concentração, percepção sobre a causa dos problemas e de progressão no alcance de metas. Esses últimos autores também sugerem o follow-up no monitoramento dos efeitos apresentados.

Sharplin, O'Neill e Chapman (2011) observaram que, apesar dos exercícios e do relaxamento terem sido considerados importantes 
para o coping, os professores relatavam entraves decorrentes de problemas com o tempo e com o espaço escolar. Com isso, promover intervenções com foco exclusivo em habilidades de coping seria útil, porém limitado em função da ausência de melhorias nas condições de trabalho (LAUGAA; RASCLE; BRUCHON-SCHWEITZER, 2008). Desse modo, é necessária a expansão no investimento em espaços para o bem-estar profissional, já que as escolas parecem estar mais centradas na implementação de rotinas para o aprendizado discente do que nas necessidades dos professores de se restaurarem frente às exigências do trabalho (GULWADI, 2006).

\section{CONSIDERACִÕES FINAIS}

Esta revisão de literatura teve por objetivo analisar publicações de bases internacionais sobre indicadores de estresse e de coping em professores, considerando a importância de seu papel nas experiências de ensino e aprendizagem. Algumas dificuldades foram encontradas durante o processo de análise dos artigos. Muitos não puderam ser acessados integralmente, o que diminuiu a extensão da revisão proposta. Destaca-se também a ausência de estudos brasileiros na amostra analisada, o que aponta a necessidade de maior abrangência dos resultados atingidos pelas pesquisas nacionais.

Além disso, seria necessária uma revisão da literatura nacional dos últimos anos sobre o assunto, para maior clareza sobre os estressores mais comumente percebidos entre professores brasileiros, quais seus níveis de estresse e, especialmente, quais as estratégias de enfrentamento empregadas. Igualmente importante seria analisar o papel de variáveis como a disponibilidade de recursos formativos e de suporte para a realização do trabalho cotidiano.

As interpretações sobre as relações encontradas entre as variáveis devem ser cautelosas para se evitarem generalizações. Isso porque os estudos contaram com diferentes composições amostrais, representadas por diferentes proporções quanto à idade, sexo, modalidade de ensino, tempo de serviço, entre outras características.

Ainda sobre o método dos estudos, percebeu-se a prevalência de estudos correlacionais aplicados em amostras relativamente grandes e acompanhados de análise quantitativa. Tais propriedades permitem a análise de relações entre diferentes variáveis em contextos 
específicos, com consequente proposição de modelos teóricos, sejam causais, mediadores ou moderadores (COZBY, 2003; CRESWELL, 2007). Entretanto, percebe-se a necessidade de estudos qualitativos para o desenvolvimento da compreensão sobre as percepções de eventos estressantes no trabalho e as estratégias de enfrentamento utilizadas em decorrência desses eventos.

Não foram registrados estudos que observassem o comportamento manifesto no ambiente escolar. Tais estudos são importantes quando se deseja avaliar os reais impactos das condições de estresse vivenciadas pelos professores nas experiências em sala de aula. Julga-se necessário, então, investigar possíveis diferenças no manejo da turma, principalmente no que diz respeito à estimulação da motivação dos alunos quanto ao processo de aprendizagem, considerando, para tanto, a presença de estresse docente. As respostas frente aos estressores em sala de aula (como o comportamento disruptivo dos alunos) podem ser avaliadas a partir desses estudos, possibilitando, em segundo plano, a inferência sobre as respostas de coping.

Este sofre influência de diferentes fatores, como o tempo de docência, o local e as condições de trabalho, o gênero, idade, estado civil, além da autoeficácia, confirmando outros estudos na área (CARLOTTO; CÂMARA, 2008; GOMES; PEREIRA, 2008; POCINHO; CAPELO, 2009). Destaca-se a diversidade nas medidas e nas dimensões avaliadas, apesar de muitos estudos terem contato com instrumentos de base transacional para a avaliação do coping. Em contrapartida à diversidade encontrada e à cautela na generalização e na uniformização dos resultados, os instrumentos descritos podem ser analisados em sua adequação para futuras pesquisas.

Os dados encontrados reforçam que os professores constituem uma classe profissional bastante exposta ao estresse. Também indicam que, muitas vezes, o excesso de demandas no trabalho pode exceder as habilidades pessoais de enfrentamento de problemas e de manejo do estresse. Além disso, muitos docentes têm de conciliar as demandas do ambiente escolar com suas demandas pessoais ou familiares, tornando o trabalho mais exaustivo (ROSS; ALTMAIER, 1994).

A partir da análise realizada, considera-se que o estresse e o coping podem afetar os processos de ensino. As estratégias de enfrentamento utilizadas para lidar com as situações estressantes, tanto na escola quanto na sua vida pessoal, podem influenciar a forma com 
que os professores lidarão com os alunos em sala de aula, bem como a administração dos recursos didáticos (ANTONIOU; POLYCHRONI; KOTRONI, 2009; CARMONA et al., 2006; CHAN, 2008).

Com isso, a organização de intervenções com foco na administração de situações de estresse frente às exigências escolares pode ser um recurso útil. Entende-se que o suporte para a promoção de estratégias de enfrentamento no processo de ensino pode aprimorar a percepção de autoeficácia e também os indicadores de saúde ocupacional. Com isso, a qualidade do ensino destinado pode acentuar-se ainda mais. É importante, também, que se considere a possibilidade de diferentes tipos de intervenções, em diferentes níveis de prevenção e promoção (NATIONAL RESEARCH COUNCIL AND INSTITUTE OF MEDICINE, 2009), a partir de técnicas direcionadas às mudanças no ambiente de trabalho, à reorganização institucional, ao desenvolvimento de habilidades individuais e à manifestação de recursos de coping que sejam adequados às diferentes realidades de trabalho e às demandas existentes.

\section{REFERÊNCIAS}

ABIDIN, R. R.; GREENE, R. W.; KONOLD, T. K. Index of teaching stress professional manual. Lutz: Psychological Assessment Resources, Inc, 2004.

ALDWIN, C. M. Stress, coping, and development. an integrative perspective. New York: Guilford Press, 2007.

ALMOG, O.; SHECHTMAN, Z. Teachers' democratic and efficacy beliefs and styles of coping with behavioural problems of pupils with special needs. European Journal of Special Needs Education, v. 22, n. 2, p. 115-129, 2007.

ALVES, M. N.; OLIVEIRA, A. M. O Efeito do desemprego no estresse e coping dos professores do $2^{\circ}$ ciclo. Psicologia, Saúde \& Doenças, Lisboa, v. 9, n. 2, p. 335-347, 2008.

ANTONIOU, A. S; POLYCHRONI, F.; KOTRONI, C. Working with students with special educational needs in Greece: teachers' stressors and coping strategies. International Journal of Special Education, v. 24, n. 1, p. 100-111, 2009.

BALOGLU, N. The relationship between prospective teachers' strategies for coping with stress and their perceptions of student control. Social Behavior and Personality, v. 36, n. 7, p. 903-910, 2008.

BANDURA, A. Self-efficacy: the exercise of control. New York: W. H. Freeman, 1997.

BENEVIDES-PEREIRA, A. M. T. et al. Sintomas de estresse em educadores brasileiros, Aletheia, Canoas, v. 17, p. 63-72, 2003.

BENEVIDES-PEREIRA, A. M. T. Burnout: quando o trabalho ameaça o bem-estar do trabalhador. São Paulo: Casa do Psicólogo, 2002. 
BOHM-KASPER, O.; et al:: Das Erfurter Belastungs-Inventar zur Erfassung von Belastung und Beanspruchung von Lehrern und Schu "lern am Gymnasium [The Erfurt Stress Inventory for the assessment of stress and strain in teachers and students at upper track high schools]. In MERKENS, H.; WEISHAUPT, H. (Eds.). Schulforschung und Schulentwicklung: Aktuelle Forschungsbeitra. Erfurt, Germany: University of Erfurt, 2000. pp. 78-80.

BOYD, N.; LEWIN, J. E.; SAGER, J. K. A model of stress and coping and their influence on individual and organizational outcomes. Journal of Vocational Behavior, v. 75, p. 197-211, 2009.

CARDOSO, J. P. et al.. Prevalência de dor musculoesquelética em professores. Revista Brasileira de Epidemiologia, São Paulo, v. 12, n. 4, p. 604-614, 2009.

CARLOTTO, M. S.; CÂMARA, S. G. Síndrome de Burnout e estratégias de enfrentamento em professores de escolas públicas e privadas. Psicologia da Educação, São Paulo, v. 26, p. 29-46, 2008.

CARMONA, C. et al. Do social comparison and coping styles play a role in the development of burnout? Cross-sectional and longitudinal findings. Journal of Occupational and Organizational Psychology, v. 79, p. 85-99, 2006.

CARVER, C. S.; SCHEIER, M. F.; WEINTRAUB, J. K. Assessing coping strategies: a theoretically based approach. Journal of Personality and Social Psychology, v. 56, p. 267-283, 1989.

CHAN, D.W. Emotional intelligence, self-efficacy, and coping among Chinese prospective and in-service teachers in Hong Kong. Educational Psychology, v. 28, n. 4, p. 397-408, 2008.

CHANG, M. L. Teacher emotion management in the classroom: appraisals, regulation, and coping with emotions. American Educational Research Association, San Diego, p. 1-12, 2009.

COMPAS, B. E. Psychobiological processes of stress and coping: implications for resilience in children and adolescents. Comments on the papers of Romeo \& McEwen and Fisher et al. Annals of the New York. Academy of Sciences, v. 1094, p. 226-234, 2006.

COOPER, C. L.; SLOAN, S. J.; WILLIAMS, S. Occupational stress Iindicator management guide. Windsor: NFER-Nelson, 1988.

COZBY, P. C.Métodos de pesquisa em ciências do comportamento. São Paulo: Atlas, 2003.

CRESWELL, J. W. Projeto de Pesquisa: métodos qualitativo, quantitativo e misto. 2. ed. Porto Alegre: Artmed, 2007.

DELCOR, N. S. et al. Condições de trabalho e saúde dos professores da rede particular de ensino de Vitória da Conquista, Bahia, Brasil. Cadernos de Saúde Pública, Rio de Janeiro, v. 20, n. 1, p. 187-196, 2004.

DOMÉNECH-BETORET, F. Stressors, self-efficacy, coping resources and burnout among secondary school teachers in Spain. Educational Psychology, v. 26, p. 519-539, 2006.

DOMÉNECH- BETORET, F.; ARTIGA, A. G. Barriers perceived by teachers at work, coping strategies, self-efficacy and burnout. The Spanish Journal of Psychology, v. 13, n. 2, p. 637-654, 2010.

EVERAERT, H. A. Het meten van de meester (Measuring Teacher Stress). In VAN DER WOLF, K. (Ed.). Het hoofd van de meester (pp. 33-61). Utrecht: Uitgeverij Agiel, 2003.

FOLKMAN, S.; LAZARUS, R. S. Manual for the ways of coping questionnaire. Palo Alto, CA: Consulting Psychologists Press, 1988.

GASPARINI, S. M.; BARRETO, S. M.; ASSUNÇÃO, A. A. O professor, as condições de trabalho e os efeitos sobre sua saúde. Educação e Pesquisa, São Paulo, v. 31, n. 2, p. 189-199, 2005. 
GOLD, E. et al. Mindfulness-based stress reduction (MBSR) for primary school teachers. Journal of Child and Family Studies, v. 19, p. 184-189, 2010.

GOMES, R. M. S.; PEREIRA, A. M. S. Estratégias de coping em educadores de infância portugueses. Psicologia Escolar e Educacional, Campinas, v. 12, n. 2, p. 319-326, 2008.

GOULART JUNIOR, E.; LIPP, M. E. N. Estresse entre professoras do Ensino Fundamental de escolas públicas estaduais. Psicologia em Estudo, Maringá, v. 13, n. 4, p. 847-857, 2008.

GULWADI, G.B. Seeking restorative experiences: elementary school teachers' choices for places that enable coping with stress. Environment and Behavior, v. 38, n. 4, p. 503-520, 2006.

HANKIN, B. L.; ABELA, J. R. Z. Development of psychopathology: A vulnerability--stress perspective. Thousand Oaks: Sage, 2005.

HENRY, D.; CRAWFORD, J. R. The short-form version of the Depression Anxiety Stress Scales (DASS-21): Construct validity and normative data in a large non-clinical sample. British Journal of Clinical Psychology, v. 44, p. 227-239, 2005.

KELLY, A. et al.Challenging behavior: principals' experience of stress and perception of the effects of challenging behavior on staff in special schools in Ireland. European Journal of Special Needs Education, v. 22, n. 2, p. 161-181, 2007.

KLUSMANN, U. et al. Engagement and emotional exhaustion in teachers: does the school context make a difference? Applied Psychology: An International Review, v. 57, p. 127-151, 2008.

KLUSMANN, U. et al. Teachers' occupational well-being and quality of instruction: the important role of self-regulatory patterns. Journal of Educational Psychology, v. 100, n. 3, p. 702-715, 2008.

LAMBERT, R. G.; MCCARTHY, C. J.; ABBOT'T-SHIM, M. Classroom appraisal of resources and demands, school-age version. Atlanta: Head Start Quality Research Center, 2001.

LAMBERT, R. G. et al. Measuring elementary teacher stress and coping in the classroom: validity evidence for the classroom appraisal of resources and demands. Psychology in the Schools, v. 46, n. 10, p. 973-988, 2009.

LAUGAA, D.; RASCLE, N.; BRUCHON-SCHWEITZER, M. Stress and burnout among French elementary school teachers: a transactional approach. Revue Européenne de Psychologie Appliquée, v. 58, p. 241-251, 2008.

LAZARUS, R. S.; FOLKMAN, S. Stress, appraisal and coping. New York: Springer Publishing Company, 1984.

LHOSPITAL, A.S.; GREGORY, A. Changes in teacher stress through participation in prereferral intervention teams. Psychology in the Schools, v. 46, n. 10, p. 1098-1112, 2009.

LIPP, M. E. N. O modelo quadrifásico do stress. In: LIPP, M. E. N. (Ed.). Mecanismos neuropsicofisiológicos do stress: teoria e aplicações clínicas.. São Paulo: Casa do Psicólogo, 2003. p. 17-22.

LIPP, M. E. N. O estresse do professor. Campinas: Papirus, 2002.

LUCHESI, K. F. et al. Problemas vocais no trabalho: prevenção na prática docente sob a óptica do professor. Saúde e Sociedade, São Paulo, v.18, n. 4, p. 673-681, 2009.

MASLACH, C.; JACKSON, S. E. Maslach Burnout Inventory (2. ed). Palo Alto: Consulting Psychologist Press, 1986. 
MOREIRA, W. Revisão de Literatura e Desenvolvimento Científico: conceitos e estratégias para confecção. Janus, Lorena, v. 1, n. 1, p. 19-31, 2004.

MURTA, S. G.; TRÓCCOLI, B. Avaliação de intervenção em estresse ocupacional. Psicologia: Teoria e Pesquisa, Brasília, v. 20, p. 39-47, 2004.

NATIONAL RESEARCH COUNCIL AND INSTITUTE OF MEDICINE. Defining the scope of prevention. In: Preventing mental, emotional, and behavioral disorders among young people: progress and possibilities. Committee on the Prevention of Mental Disorders and Substance Abuse Among Children, Youth, and Young Adults: Research Advances and Promising Interventions. Washington, DC: The National Academies Press, 2009. pp. 59-69.

POCINHO, M.; CAPELO, M. R. Vulnerabilidade ao stress, estratégias de coping e autoeficácia em professores portugueses. Educação e Pesquisa, São Paulo, v. 35, n. 2, p. 351-367, 2009.

RIEG, S.A.; PAQUETTE, K.R.; CHEN, Y. Coping with stress: an investigation of novice teachers' stressors in the elementary classroom. Education, v. 128, n. 2, p. 211-226, 2007.

ROSS, R.; ALTMAIER, E. Intervention in occupational stress. London: Thousand Oaks \& New Delhi, Sage Publications, 1994.

SCHAARSCHMIDT, U.; FISCHER, A. AVEM. Ein diagnostisches Instrument zur Differenzierung von Typen gesundheitsrelevanten Verhaltens und Erlebens gegenüber der Arbeit [AVEM-An instrument for diagnosing different types of work- and health-related behavior and experience]. Zeitschrift für Differentielle und Diagnostische Psychologie, v. 18, p. 151-163, 1997.

SELYE, H. The stress concept: Past, present and future. In: COOPER, C. L. (Ed.), Stress research: issues for the eighties. Chichester: John Wiley \& Sons, 1983. p. 1-20.

SHARPLIN, E.; O’NEILL, M.; CHAPMAN, A. Coping strategies for adaptation to new teacher appointments: intervention for retention. Teaching and Teacher Education, v. 27, p. 136-146, 2011.

SHEN, Y. E. Relationships between self-efficacy, social support and stress-coping strategies in Chinese primary and secondary school teachers. Stress and Health, v. 25, p. 129-138, 2009.

SKINNER, E. A. et al. Searching for the structure of coping: a review and critique of category systems for classifying ways of coping. Pychological Bulletin, v.129, n. 2, p. 216-269, 2003.

SKINNER, E. A.; ZIMMER-GEMBECK, M. J. The development of coping. Annual Revien of Psychology, v. 58, p. 119-144, 2007.

STOEBER, J.; RENNERT, D. Perfectionism in school teachers: relations with stress appraisals, coping styles, and burnout. Anxiety, Stress, \& Coping, v. 21, n. 1, p. 37-53, 2008.

STRAUSS, A.; CORBIN, J. Basics of qualitative research: grounded procedures and techniques (2 ed.). Thousand Oaks, CA: Sage, 1998.

TRAVERS, C.; COOPER, C. Teachers under p: stress in the teaching profession. London: Routledge, 1996.

VEDOVATO, T. G.; MONTEIRO, M. I. Perfil sócio-demográfico e condições de saúde e trabalho dos professores de nove escolas estaduais paulistas. Revista Escola de Enfermagem, São Paulo, v. 42, n. 2, p. 291-297, 2008.

WITTER, G. P. Professor-estresse: análise de produção científica. Psicologia Escolar e Educacional, Campinas, v. 7, n. 1, p. 1-17, 2003.

WONG, K. S.; WAI, H. C.; ROSEN, S. Experience of being spurned: coping style, stress preparation, and depersonalization in beginning kindergarten teachers. Journal of Research in Childhood Education, v. 22, n.2, p. 141-170, 2007. 
YAEGASHI, S. F. R.; .et. al. O trabalho docente e o burnout. um estudo com professores paranaenses. In: VIII Congresso Nacional de Educação - EDUCERE, 2008, Curitiba. Anais do VIII Congresso Nacional de Educação - EDUCERE. Curitiba : Champagnat, 2008. v. 1. p. 4870-4884.

ZAFFARI, N.T. et al. Síndrome de burnout e estratégias de coping em professores: diferença entre gêneros. Revista de Psicologia IESB-Instituto de Educação Superior de Brasília, Brasília, v. 1, n. 2, p. 1-12, 2009

ZURLO, M. C.; PES, D.; COOPER, C. L. Stress in teaching: a study of occupational stress and its determinants among Italian school teachers. Stress and Health, v. 23, p. 231-241, 2007.

Recebido: $25 / 10 / 2012$

Aprovado: 19/05/2014

Contato:

Av. Fernando Ferrari, 514 Goiabeiras

Vitória | ES | Brasil

CEP 29.075-910 